\title{
Distribution of Lymph Nodes in Stage III Patients With Mid and Low Rectal Cancer: Preliminary Study
}

\author{
Sohyun Kim \\ Department of Surgery, Yeungnam University College of Medicine, Daegu, Korea
}

Purpose: Most patients with rectal cancer undergo a total mesorectal excision and a partial resection of the sigmoid colon to improve oncologic outcomes. The aim of this study was to assess the distribution of lymph nodes (LNs) in rectal cancer. Methods: The records of 54 patients with mid and low rectal cancer between April 2015 and March 2017 were reviewed, and 49 patients were enrolled in this study. All harvested LNs were analyzed according to the harvested area: the mesorectum area (MA), the vascular pedicle area (VA), and the sigmoid area (SA).

Results: Finally, 865 LNs were harvested from all patients, and of these, $71(8.2 \%)$ showed metastases. In stage III patients, $343 \mathrm{LNs}$ were harvested, and of these, $52(15.2 \%)$ showed metastases. Significant differences were found in the total numbers of harvested LNs by area $(\mathrm{P}<0.001)$ and in the numbers of harvested positive LNs by area $(\mathrm{P}<0.001)$. In stage III patients, LNs from the MA were more frequently to be positive than were those from the VA $(\mathrm{P}<0.001)$ or the SA $(\mathrm{P}<$ $0.001)$.

Conclusion: LN metastasis in the SA was rare. Therefore, resecting the mesorectum and the vascular pedicle may be more important than resecting the sigmoid mesentery due to concerns about LN metastases.

Keywords: Rectal neoplasm; Lymph node; Mesorectum; Distribution

\section{INTRODUCTION}

Rectal cancer surgery requires several sophisticated skills to improve oncologic outcomes. Patients with rectal cancer undergo removal of the primary tumor with adequate resection margin and regional lymphadenectomy [1], and the lymphadenectomy is determined by blood supply and lymphatic system drainage $[1,2]$. Thus, ligating the inferior mesenteric artery (IMA) close to the aorta and a total mesorectal excision are standard procedures in rectal cancer [3]. Specimens of rectal cancer contained the rectum with mesorectum, the IMA, superior rectal artery pedicles, and

Received: October 31, 2017 - Accepted: November 27, 2017

Correspondence to: Sohyun Kim, M.D.

Department of Surgery, Yeungnam University Medical Center, Yeungnam

University College of Medicine, 170 Hyeonchung-ro, Nam-gu, Daegu 42415, Korea

Tel: +82-53-620-3594, Fax: +82-53-624-1213

E-mail: kshgs@yu.ac.kr

ORCID code: https://orcid.org/0000-0002-8625-329X

(C) 2018 The Korean Society of Coloproctology

This is an open-access article distributed under the terms of the Creative Commons Attribution NonCommercial License (http://creativecommons.org/licenses/by-nc/4.0) which permits unrestricted noncommercial use, distribution, and reproduction in any medium, provided the original work is properly cited. part of the sigmoid colon [4].

Continuity of the gastrointestinal tract after rectal cancer surgery is an important issue. More patients undergo a low anterior or an intersphincteric resection than undergo an abdominoperineal resection [5]. Anastomotic integrity entails a sufficiently long colon and good blood perfusion. Many surgeons practice high ligation of the IMA and splenic mobilization to acquire tensionfree anastomosis $[3,6]$. However, these procedures remove long lengths of the sigmoid colon. High ligation of the IMA compromises perfusion of anastomosis in patients with cardiovascular problems [7]. On the other hands, low ligation of the IMA provides a shorter colon length for anastomosis than high ligation of the IMA $[8,9]$. The specimen length of the IMA has been reported to be shorter for low ligation than for high ligation of IMA [9]. The author of this study wondered whether or not the part of the sigmoid colon in the rectal cancer specimens was important for improving oncologic outcomes and wondered how many lymph nodes (LNs) were positive in the sigmoid colon. The aim of this study was to assess the distribution of LNs in rectal cancer. This study should provide understanding of how rectal LNs were distributed and patterns of metastases. 


\section{METHODS}

The records of 54 patients with mid or low rectal cancer between April 2015 and March 2017 were reviewed. The study was approved by the Institutional Review Board of the Yeungnam University Hospital. Formal consent of the patients was not required to this study. Middle rectal cancer was defined as the distal extent of the cancer occurring within 7 to $11 \mathrm{~cm}$ from the anal verge according to abdominopelvic computed tomography (CT), magnetic resonance imaging (MRI), or flexible sigmoidoscopy [10]. Low rectal cancer was defined as the distal extent of the cancer occurring within $6 \mathrm{~cm}$ from the anal verge according to CT, MRI, or flexible sigmoidoscopy [10]. The inclusion criteria were (1) a histologically proven adenocarcinoma, (2) a curative resection of the primary tumor, and (3) no history of hereditary, metachronous, or synchronous colorectal cancer or any other malignancy. Of 54 patients, 2 patients with a carcinoma in situ (Tis), 1 patient with metachronous colorectal cancer, and 2 patients with synchronous colorectal cancer were excluded. Finally, 49 patients were enrolled in this study after pathologic testing. To access the tendency of LN metastases, 20 patients with stage III cancer were analyzed separately. On the basis of established preoperative studies, a portion of the patients underwent neoadjuvant long-course chemoradiotherapy with concurrent infusional 5-fluorouracil or oral capecitabine; the patients who received chemoradiotherapy underwent surgery 6-10 weeks later. All patients underwent the standard procedure a total mesorectal excision using a no-touch isolation technique. In the patients who underwent low ligation of the IMA, lymphatic tissues were gathered from the remnants of the proximal IMA.

After surgery, the surgeons painted all specimens with inks, separated the tissue around the proximal IMA and the superior rectal artery from the specimen, and referred to this tissue as the vascular pedicle area (VA; Fig. 1). The sigmoid mesentery was defined as the distal extent of the marginal artery of Drummond, and the surgeons referred to this tissue as the sigmoid area (SA; Fig. 1). The distal parts of the specimens except for the sigmoid colon were the mesorectum and were referred to as the mesorectum area (MA; Fig. 1). Surgeons measured the length of each area. All specimens were processed by a single pathologist and fixed in formalin for 48-72 hours. All LNs were harvested by using a meticulous manual dissection without fat clearance, and all were labeled according to the harvested zone.

All data were analyzed using IBM SPSS Statistics ver. 23.0 (IBM Co., Armonk, NY, USA). Continuous data were analyzed with the Student t-test. The harvested and the positive LNs were analyzed using the Kruskal-Wallis test or the Mann-Whitney tests or analysis of variance. The harvested LNs and the specimen length and the positive LNs and specimen length were analyzed using Spearman correlation coefficient. Statistical significance was established at P-value less than 0.05 .

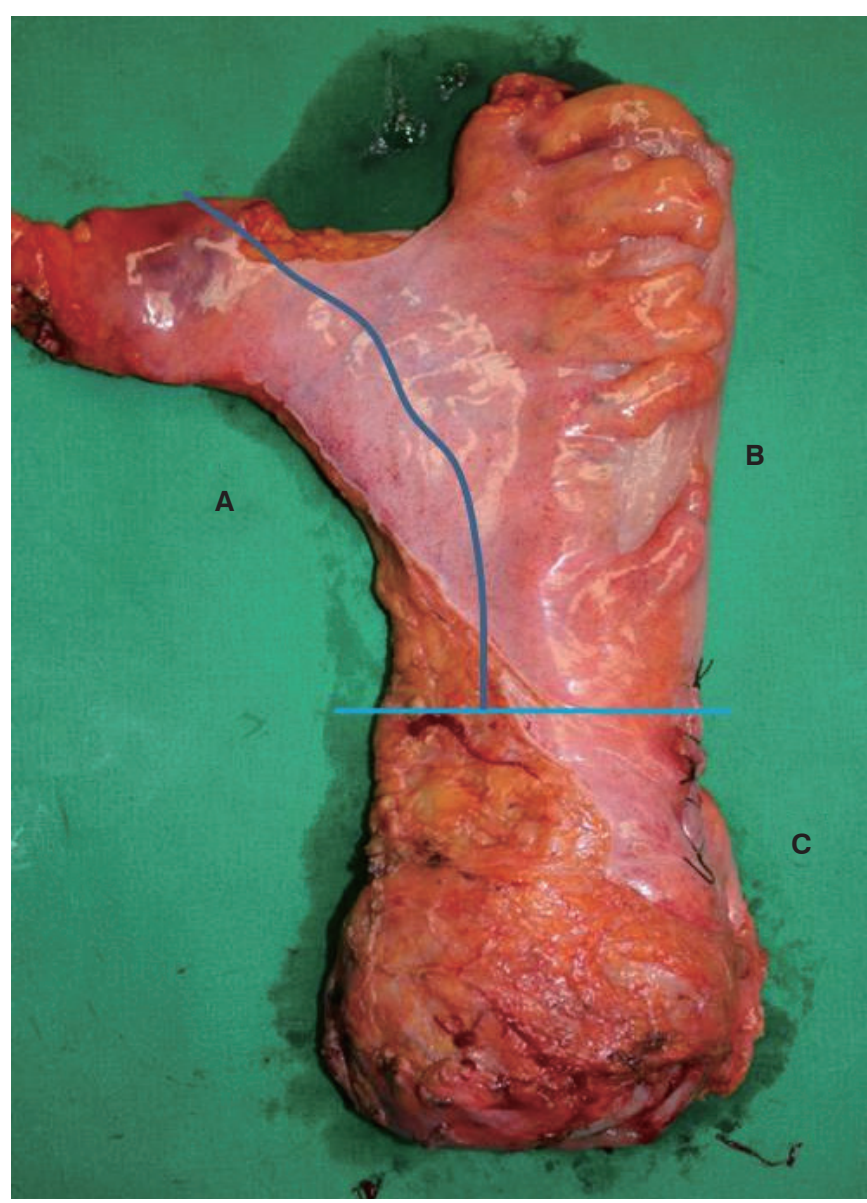

Fig. 1. Areas of a harvested lymph node: vascular pedicle area (A), sigmoid area (B), and mesorectum area (C).

\section{RESULTS}

This study 49 patients ( 28 male, 21 female patients), with a mean age of 65 years (range, $40-85$ years). Of the 49 patients, 42 underwent a low anterior resection and 7 underwent an abdominoperineal resection. The clinicopathologic characteristics of all patients and of stage III patients are described in Table 1. The median length of the proximal margin was $13.1 \mathrm{~cm}$ (range, $4.3-31.5 \mathrm{~cm}$ ), and the median length of the distal margin was $2.0 \mathrm{~cm}$ (range, $0.7-5.0 \mathrm{~cm}$ ).

Finally, 865 LNs were harvested from all specimens, and of these, $71(8.2 \%)$ showed metastases. The mean total $\mathrm{LN}$ and positive LN counts were $17.65 \pm 1.27$ and $1.45 \pm 0.27$, respectively. The mean total harvested LN counts are showed by area in Table 2. Of the $865 \mathrm{LNs}, 30.5 \%$ were in the VA, $21.2 \%$ in the SA, and $48.3 \%$ in the MA; among the positive LNs, $11.3 \%$ were in the VA, $0.6 \%$ in the SA, and $81.7 \%$ in the MA.

In stage III patients, $343 \mathrm{LNs}$ were harvested from specimens, and of these, 52 (15.2\%) showed metastases. The median total LN and positive LN counts were 15.50 and 2.50, respectively. The me- 
Table 1. Clinicopathologic characteristics of the patients

\begin{tabular}{|c|c|c|}
\hline Characteristic & $\begin{array}{l}\text { All patients } \\
\quad(n=49)\end{array}$ & $\begin{array}{l}\text { Stage III patients } \\
\qquad(\mathrm{n}=20)\end{array}$ \\
\hline \multicolumn{3}{|l|}{ Sex } \\
\hline Male & $28(57.1)$ & $11(55.0)$ \\
\hline Female & $21(42.9)$ & $9(45.0)$ \\
\hline Age (yr) & & $69(50-85)$ \\
\hline \multicolumn{3}{|l|}{ Neoadjuvant chemoradiotherapy } \\
\hline Yes & $13(26.5)$ & $4(20.0)$ \\
\hline No & $36(73.5)$ & $16(80.0)$ \\
\hline \multicolumn{3}{|l|}{ ASA PS classification } \\
\hline $1-2$ & $41(83.7)$ & $15(75.0)$ \\
\hline$\geq 3$ & 8 (16.3) & $5(25.0)$ \\
\hline \multicolumn{3}{|l|}{ Operation } \\
\hline Open & $9(18.4)$ & $5(25.0)$ \\
\hline Laparoscopic & $40(81.6)$ & $15(75.0)$ \\
\hline \multicolumn{3}{|l|}{ Tumor location } \\
\hline Middle rectum & $26(53.1)$ & $11(55.0)$ \\
\hline Low rectum & $23(46.9)$ & $9(45.0)$ \\
\hline \multicolumn{3}{|l|}{ Colon resection } \\
\hline LAR & $42(85.7)$ & $17(85.0)$ \\
\hline APR & 7 (14.3) & $3(15.0)$ \\
\hline \multicolumn{3}{|l|}{ IMA ligation } \\
\hline High & $29(59.2)$ & $10(50.0)$ \\
\hline Low & $20(40.8)$ & $10(50.0)$ \\
\hline Size of tumor (cm) & $4.94(1.5-11.5)$ & $5.25(1.7-11.5)$ \\
\hline Length of proximal margin (cm) & $12.90(4.3-31.5)$ & $14.25(6.0-31.5)$ \\
\hline Length of distal margin (cm) & $2.01(0.07-5.0)$ & $1.65(0.7-5.0)$ \\
\hline \multicolumn{3}{|l|}{ Cell differentiation } \\
\hline Well & $1(2.0)$ & $0(0)$ \\
\hline Moderate & $43(87.8)$ & $18(90.0)$ \\
\hline Poorly & 5 (10.2) & $2(10.0)$ \\
\hline \multicolumn{3}{|l|}{ Lymphatic invasion } \\
\hline Yes & 26 (53.1) & $15(75.0)$ \\
\hline No & 23 (46.9) & $5(25.0)$ \\
\hline \multicolumn{3}{|l|}{ Vascular invasion } \\
\hline Yes & $13(26.5)$ & $8(40.0)$ \\
\hline No & 36 (73.5) & $12(60.0)$ \\
\hline \multicolumn{3}{|l|}{ Neural invasion } \\
\hline Yes & $18(36.7)$ & $9(45.0)$ \\
\hline No & 31 (63.3) & $11(55.0)$ \\
\hline \multicolumn{3}{|l|}{ T stage } \\
\hline 1 & $5(10.2)$ & $2(10.0)$ \\
\hline 2 & $5(10.2)$ & $0(0)$ \\
\hline 3 & 36 (73.5) & $16(80.0)$ \\
\hline 4 & $3(6.1)$ & $2(10.0)$ \\
\hline
\end{tabular}

(Continued to the next)
Table 1. Continued

\begin{tabular}{lcc}
\hline Characteristic & $\begin{array}{c}\text { All patients } \\
(\mathrm{n}=49)\end{array}$ & $\begin{array}{c}\text { Stage III patients } \\
(\mathrm{n}=20)\end{array}$ \\
\hline $\mathrm{N}$ stage & & \\
0 & $23(46.9)$ & $0(0)$ \\
$1 \mathrm{a}$ & $7(14.3)$ & $8(40.0)$ \\
$1 \mathrm{~b}$ & $11(22.4)$ & $7(35.0)$ \\
$1 \mathrm{c}$ & $1(2.0)$ & $0(0)$ \\
\hline $\mathrm{a}$ & $6(12.2)$ & $5(25.0)$ \\
\hline $\mathrm{b}$ & $1(2.0)$ & $0(0)$ \\
\hline
\end{tabular}

Values are presented as number (\%) or median (range).

ASA PS, American Society of Anesthesiologists physical status; LAR, low anterior resection; APR, abdominoperineal resection; IMA, inferior mesenteric artery.

Table 2. Mean number of lymph nodes (LNs) and median number of LNs of stage III patients

\begin{tabular}{lcc}
\hline Lymph node & All patients $(\mathrm{n}=49)$ & Stage III patients $(\mathrm{n}=\mathbf{2 0})$ \\
\hline Total LN & 865 & 343 \\
No. of total LNs & $17.65 \pm 1.27$ & 15.5 \\
No. LNs of VA & $5.39 \pm 0.52$ & 5.0 \\
No. LNs of SA & $3.73 \pm 0.43$ & 2.5 \\
No. LNs of MA & $8.53 \pm 0.86$ & 9.0 \\
\hline
\end{tabular}

Values are presented as mean \pm standard deviation.

VA, vascular pedicle area; SA, sigmoid area; MA; mesorectum area.

Table 3. Distribution of the total number of lymph nodes

\begin{tabular}{|c|c|c|}
\hline \multirow{2}{*}{ Area } & All patient $(n=49)$ & Stage III patients $(n=20)$ \\
\hline & P-value & P-value \\
\hline \multicolumn{3}{|l|}{$\overline{V A}$} \\
\hline SA & 0.830 & 0.046 \\
\hline MA & $<0.001$ & 0.018 \\
\hline \multicolumn{3}{|l|}{ SA } \\
\hline MA & $<0.001$ & $<0.001$ \\
\hline
\end{tabular}

VA, vascular pedicle area; SA, sigmoid area; MA, mesorectum area.

dian total harvested LN counts are presented in Table 2. By percentage and area, of the 343 harvested LNs, $30.9 \%$ were in the VA, $17.2 \%$ in the SA, and $51.9 \%$ in the MA; among the positive LNs, $7.7 \%$ were in the VA, $1.9 \%$ in the SA, and $90.4 \%$ in the MA.

The LN distribution by area was analyzed, and significant differences were found in the total harvested LNs according to area (P $<0.001)$. More LNs were harvested from the MA than the VA (P $<0.001)$ and the SA $(\mathrm{P}<0.001)$ (Table 3). However, the difference in total harvested LNs between the VA and the SA was not significant $(\mathrm{P}=0.083)$. Nevertheless, some of the differences in harvested LNs from among the 3 areas were significant $(\mathrm{P}<0.001)$. LNs in the MA were more frequently positive than those in the 
Table 4. Distribution of the total number of positive lymph nodes

\begin{tabular}{|c|c|c|}
\hline \multirow{2}{*}{ Area } & All patient $(n=49)$ & Stage III patients $(n=20)$ \\
\hline & P-value & P-value \\
\hline \multicolumn{3}{|l|}{ VA } \\
\hline SA & 0.018 & 0.157 \\
\hline MA & 0.007 & $<0.001$ \\
\hline \multicolumn{3}{|l|}{ SA } \\
\hline MA & $<0.001$ & $<0.001$ \\
\hline
\end{tabular}

VA, vascular pedicle area; SA, sigmoid area; MA, mesorectum area.

VA $(\mathrm{P}=0.007)$ or in the SA $(\mathrm{P}<0.001)$. Moreover, a significant difference in positive harvested LNs between the VA and the SA was observed $(\mathrm{P}=0.018)$ (Table 4$)$.

In stage III patients, LN distribution by area was analyzed, and significant differences in the total harvested LNs were found according to the area $(\mathrm{P}<0.001)$. More LNs were harvested from the VA than from the SA $(\mathrm{P}=0.046)$, and more were harvested from the MA than from the SA $(\mathrm{P}<0.001)$ or the VA $(\mathrm{P}=0.018)$ (Table 3). For positive LNs, significant differences were found among the three areas $(\mathrm{P}<0.001)$. LNs in the MA were more frequently positive than those in the VA $(\mathrm{P}<0.001)$ or in the SA $(\mathrm{P}$ $<0.001)$. However, no significant differences in positive harvested LNs between the VA and the SA were found $(\mathrm{P}=0.157)$ (Table 4).

The median lengths of the MA and the SA were $8.7 \mathrm{~cm}$ (range, $6.0-15.0 \mathrm{~cm}$ ) and $11.3 \mathrm{~cm}$ (range, $5.0-23.0 \mathrm{~cm}$ ), respectively, and the median length of the VA was $11.6 \mathrm{~cm}$ (range, $9.0-15.0 \mathrm{~cm}$ ). No significant correlation was found between the MA length and the total harvested LNs $(\mathrm{P}=0.872)$ or between the SA length and the total harvested LNs $(\mathrm{P}=0.865)$. Total harvested LNs in the VA showed no significant correlation with the VA length $(\mathrm{P}=0.188)$.

\section{DISCUSSION}

In mid and low rectal cancer, significantly more total LNs were harvested from the MA and from the VA than from the SA, and positive LNs were more frequently found in the MA than in the other areas. LN metastases of the mesorectum are extremely likely in patients with mid and low rectal cancer, and LNs in the sigmoid mesentery are unlikely. In mid and low rectal cancer, LNs in the sigmoid colon mesentery might have little oncologic effect. Most LN metastases are found in the mesorectum, and the spread of lymphatics in the rectum occurs in three directions: downward, lateral and upward [11]. In locally advanced rectal cancer, 70\%$80 \%$ of the patients show positive LNs in the mesorectum [12-14]. In the present study, all patients showed positive LNs in the mesorectum; in fact, $90 \%$ of positive LNs were located in the mesorectum. Accurate excision of the mesorectum was important for evaluating the $\mathrm{N}$ stage and improving oncologic outcomes. LNs in the mesorectum play the most important role in mid and low rectal cancer.
Less LN metastasis was found in the vascular pedicle and the sigmoid mesentery than in the mesorectum. Proximal LN metastases above the mesorectum present in $10 \%-20 \%$ of patients with rectal cancers $[12,13]$, and $23 \%$ of the LNs are located in the trunk of the superior rectal artery [13]. Langman et al. [14] reported $1.9 \%$ positive LNs in the vascular pedicle and $0.03 \%$ in the sigmoid mesentery. For all positive LNs, $16.5 \%$ and $0.3 \%$ were in the vascular pedicle and the sigmoid mesentery, respectively [14]. In most other studies, the authors did not distinguish between LNs in the vascular pedicle and LNs in the sigmoid colon mesentery, and in the present study, the rates of positive LNs in the vascular pedicle and the sigmoid mesentery for patients with stage III rectal cancer were low. In the sigmoid mesentery, 1.9\% of LNs showed metastases; thus, LNs in the sigmoid mesentery might be of limited importance in patients with rectal cancer.

Current guidelines recommend sampling at least 12 LNs for reliable LN staging in patients with colorectal cancer. However, no recommendations regarding the locations of the LNs to be harvested are available. Most LNs in this study were distributed in the mesorectum. Mesorectal LNs in patients with rectal cancer comprise over $60 \%$ of total harvested LNs, and LNs in the proximal vascular pedicle comprise over $15 \%$ of total harvested LNs [13]. In another study, the LN distribution was $40 \%$ in the mesorectum, $32 \%$ in the sigmoid mesentery, and $28 \%$ in the vascular pedicle [14]. In this study, the distribution was $52 \%$ in the mesorectum, $30 \%$ in the vascular pedicle, and $17.2 \%$ in the sigmoid mesentery. Accurate $\mathrm{N}$ staging may require targeting areas contain lymph nodes.

This study has several limitations. First, it was a retrospective study. Second, previous reports demonstrated that preoperative chemoradiotherapy influenced lymph node status in patients with rectal cancer $[15,16]$, but this study's findings did not reflect that because so few enrolled patients had been enrolled. Large-scale prospective studies are needed for evaluating LN distribution in patients with rectal cancer.

Most lymph nodes in patients with rectal cancer are located in the mesorectum or the vascular pedicle, and positive lymph nodes are more likely to be found in the mesorectum. In this study, fewer harvested and positive LNs were harvested from the sigmoid area than from the other areas. Therefore, due to the concern about LN metastases, resecting the mesorectum and the vascular pedicle may be more important than resecting the sigmoid mesentery.

\section{CONFLICT OF INTEREST}

No potential conflict of interest relevant to this article was reported.

\section{REFERENCES}

1. Schmoll HJ, Van Cutsem E, Stein A, Valentini V, Glimelius B, 
Haustermans K, et al. ESMO Consensus Guidelines for management of patients with colon and rectal cancer. a personalized approach to clinical decision making. Ann Oncol 2012;23:2479-516.

2. Nelson H, Petrelli N, Carlin A, Couture J, Fleshman J, Guillem J, et al. Guidelines 2000 for colon and rectal cancer surgery. J Natl Cancer Inst 2001;93:583-96.

3. Kanemitsu Y, Hirai T, Komori K, Kato T. Survival benefit of high ligation of the inferior mesenteric artery in sigmoid colon or rectal cancer surgery. Br J Surg 2006;93:609-15.

4. Wibe A, Møller B, Norstein J, Carlsen E, Wiig JN, Heald RJ, et al. A national strategic change in treatment policy for rectal cancer-implementation of total mesorectal excision as routine treatment in Norway. A national audit. Dis Colon Rectum 2002;45:857-66.

5. Rullier E, Denost Q, Vendrely V, Rullier A, Laurent C. Low rectal cancer: classification and standardization of surgery. Dis Colon Rectum 2013;56:560-7.

6. Titu LV, Tweedle E, Rooney PS. High tie of the inferior mesenteric artery in curative surgery for left colonic and rectal cancers: a systematic review. Dig Surg 2008;25:148-57.

7. Boström P, Haapamäki MM, Matthiessen P, Ljung R, Rutegård J, Rutegård M. High arterial ligation and risk of anastomotic leakage in anterior resection for rectal cancer in patients with increased cardiovascular risk. Colorectal Dis 2015;17:1018-27.

8. Thum-umnuaysuk S, Boonyapibal A, Geng YY, Pattana-Arun J. Lengthening of the colon for low rectal anastomosis in a cadaveric study: how much can we gain? Tech Coloproctol 2013;17:37781.

9. Bonnet S, Berger A, Hentati N, Abid B, Chevallier JM, Wind P, et al. High tie versus low tie vascular ligation of the inferior mesen- teric artery in colorectal cancer surgery: impact on the gain in colon length and implications on the feasibility of anastomoses. Dis Colon Rectum 2012;55:515-21.

10. Salerno G, Sinnatamby C, Branagan G, Daniels IR, Heald RJ, Moran BJ. Defining the rectum: surgically, radiologically and anatomically. Colorectal Dis 2006;8 Suppl 3:5-9.

11. Morikawa E, Yasutomi M, Shindou K, Matsuda T, Mori N, Hida J, et al. Distribution of metastatic lymph nodes in colorectal cancer by the modified clearing method. Dis Colon Rectum 1994;37: 219-23.

12. Kim JS, Sohn DK, Park JW, Kim DY, Chang HJ, Choi HS, et al. Prognostic significance of distribution of lymph node metastasis in advanced mid or low rectal cancer. J Surg Oncol 2011;104:48692.

13. Zheng YC, Zhou ZG, Li L, Lei WZ, Deng YL, Chen DY, et al. Distribution and patterns of lymph nodes metastases and micrometastases in the mesorectum of rectal cancer. J Surg Oncol 2007;96: 213-9.

14. Langman G, Patel A, Bowley DM. Size and distribution of lymph nodes in rectal cancer resection specimens. Dis Colon Rectum 2015;58:406-14.

15. Baxter NN, Morris AM, Rothenberger DA, Tepper JE. Impact of preoperative radiation for rectal cancer on subsequent lymph node evaluation: a population-based analysis. Int J Radiat Oncol Biol Phys 2005;61:426-31.

16. Morcos B, Baker B, Al Masri M, Haddad H, Hashem S. Lymph node yield in rectal cancer surgery: effect of preoperative chemoradiotherapy. Eur J Surg Oncol 2010;36:345-9. 